\title{
Entrelacs
}

Cinéma et audiovisuel

Jodorowsky, d'un art à l'autre

\section{Alejandro Jodorowsky, l' « artiste multiple »}

\section{Elisabeth Pouilly}

\section{(2) OpenEdition}

\section{Journals}

Édition électronique

URL : http://journals.openedition.org/entrelacs/4626

DOI : 10.4000/entrelacs.4626

ISSN : 2261-5482

\section{Éditeur}

Éditions Téraèdre

\section{Référence électronique}

Elisabeth Pouilly, « Alejandro Jodorowsky, l' « artiste multiple » », Entrelacs [En ligne], 16 | 2019, mis en ligne le 29 octobre 2019, consulté le 05 décembre 2019. URL : http://journals.openedition.org/ entrelacs/4626 ; DOI : 10.4000/entrelacs.4626

Ce document a été généré automatiquement le 5 décembre 2019

Tous droits réservés 


\title{
Alejandro Jodorowsky, l' « artiste multiple »
}

\author{
Elisabeth Pouilly
}

1 L'entretien avec Alejandro Jodorowsky a lieu dans son appartement, à Paris, le $1^{\text {er }}$ juin 2019. Au cours de cette conversation, l'artiste va nous indiquer plusieurs livres phares de sa bibliothèque qui court sur toute la hauteur des murs de son bureau : des essais qui traitent des différentes mystiques et des sagesses orientales mais aussi des bandes dessinées. Dans un coin, une grande toile est exposée sur un chevalet : une œuvre de PascALEjandro, fusion artistique de Alejandro Jodorowsky et de sa femme, Pascale Montandon-Jodorowsky. En 2015, le musée d'art contemporain de Bordeaux (CAPC) organisait la première rétrospective générale de son œuvre foisonnante. Actuellement, à 90 ans, Jodorowsky continue à signer les scénarios de plusieurs bandes dessinées, à écrire des livres et à réaliser des films. Le prochain, intitulé Psychomagie: un art pour guérir, sortira en salles en octobre 2019 et reviendra sur une autre de ses pratiques artistiques.

ELISABETH POUILLY : Comment s'est faite votre découverte des différents arts : le théâtre, la pantomime, etc. ?

Alejandro Jodorowsky : J'ai appris à lire quand j'avais cinq ans avec un maitre qui avait un abécédaire ; ça a été le changement de ma vie. Un jour, il a disposé trois cartons qui formaient le mot espagnol «ojo» qui signifie en français « œil». Je connaissais ce mot « ojo » car il est au centre de mon nom : AlejandrOJOdorowsky ». Je pense que c'est pour cela que j'ai appris à lire tout de suite.

Découvrir en soi une qualité artistique, c'est l'histoire de ma vocation: je dessine, tout à coup je deviens un acteur. Ce sont des choses que j'avais en moi. J'ai fait de la danse, des marionnettes... À ce moment-là, c'était une découverte, comme une illumination. Après c'est la réception, qui est un choc pour les autres. Tu dois être prudent pour montrer ce que tu fais parce qu'on rigole, on te critique, on t'écrase, tout cela parce qu'essentiellement l'être humain ne veut pas changer. Lorsque tu amènes un changement, tu provoques une réaction forte. Après vient un moment où 
tu imposes ce que tu fais malgré tout. Je veux être poète... mais je suis poète ! Quand tu t'imposes, on t'accepte, et quand tu es accepté, tu peux alors passer à autre chose.

J'ai toujours réalisé des œuvres d'avant-garde. J'ai monté une centaine de pièces de théâtre au Mexique: des œuvres de Samuel Beckett, d'Eugène Ionesco, de Jean Tardieu, d'August Strinberg, d'Arthur Adamov, etc. J'ai mis en scène l'art de l'absurde, tout l'art contemporain français, parce que j'avais commencé avec le mime Marceau.

E.P. : Vous avez également créé des «éphémères paniques » au Mexique.

A.J. : Oui, et à Paris aussi j'ai fait un énorme éphémère. Ça va avec la fondation du groupe Panique en France avec Fernando Arrabal et Roland Topor. Mais c'était le résultat de mes expériences de théâtre au Mexique où le théâtre était espagnol et où je me confrontais à tous ses poncifs. Quand j'ai fait du cinéma, je me suis confronté à tous les poncifs du pire cinéma hollywoodien. Quand j'ai écrit de la poésie, je me suis retrouvé avec la pire poésie, qui écrit avec des rimes et une métrique. Or, je voulais faire quelque chose de nouveau, quelque chose qui serait un art libéré des préjugés, des imitations, de ce que font les autres... Mais surtout, je voulais arriver à faire du théâtre. Mais qu'est-ce que le théâtre ? Le théâtre n'est pas de la littérature. Être acteur, ce n'est pas être artiste, c'est être interprète. De la même manière que les chanteurs sont des interprètes. Mais l'artiste, lui, est un créatif. Il doit trouver ce qu'est l'essence de la peinture, l'essence du théâtre, l'essence de la poésie.

Je pourrais énoncer des formules, comme pour définir la poésie : «Dire le maximum de choses en un minimum de mots». Mais être artiste, ce n'est pas suivre des formules mais connaître une sorte d'illumination qui s'apparente à ce que les bouddhistes Zen désignent au travers du mot « satori »" Pendant cinq années, j'ai été l'élève d'un moine Zen qui me posait des devinettes insolubles pour lesquelles on peut mettre trois jours, trois mois, trente ans à trouver la réponse. Ce sont les « koan zen $»^{2}$, cela nous apprend à nous libérer du mental - c'est ça la solution. Les mots ne sont pas les choses. Dans la philosophie, on essaie de tout trouver avec le langage, avec un livre, mais ce n'est pas possible. L'art arrive comme un rêve, comme une illumination.

Je viens de terminer une bande dessinée. Pour ce type de travail, je collabore avec un dessinateur. Je peux lui écrire une séquence qui s'achève sur une chose impossible à résoudre : le personnage est en danger et je ne sais pas comment le sortir d'une telle situation. Arrivé à ce point, j'arrête. J'attends un, deux, trois jours, et, tout à coup, une solution me vient! Elle me vient comme un rêve: c'est l'inconscient qui me l'envoie. Un artiste ne fabrique pas son œuvre, il la reçoit depuis son inconscient. C'est en quelque sorte la partie judaïque de la création, la « kabbale » signifiant en hébreu « ce qui est reçu ».

Grâce à l'art, j'ai aboli la frontière entre le conscient et l'inconscient : je ne refuse pas l'inconscient, je le reçois, donc immédiatement et de façon automatique, il me donne la solution. Cette pratique me vient du théâtre, parce que j'ai toute une expérience artistique avec les happenings, les actes artistiques puis poétiques... Je suis ensuite passé aux actes psychomagiques, notamment pour guérir ${ }^{3}$, mais pas pour soigner les maladies car cela relèverait du charlatanisme.

E. P. : Comment se fait le passage d'un art à un autre? 
A.J. : II n'y a pas de passage, c'est très facile. J'admire les touche-à-tout, les êtres multiples, tels que Leonard de Vinci, Jean Cocteau, Pier Paolo Pasolini, le poète français René Daumal... Quand on est artiste, on est tout à la fois : on ne passe pas d'un art à un autre, c'est le même artiste qui pratique un art et un autre. On ne passe pas d'une valise à une autre valise, ou d'une forme à une autre forme ; la chose vient de l'intérieur, elle est en toi si tu t'es libéré. Bien sûr, il y a le talent. On peut pratiquer l'art avec génie, médiocrité, grossièreté, imbécillité. Si, par exemple, tu es un imbécile, tu feras un art imbécile. Or, ce n'est pas à proprement parler l'art qui est imbécile, ni l'œuvre, mais l'individu seul qui s'exprime. Tout est Un: j'ai changé de mode d'expression, purement et simplement, sans changer de véhicule.

\section{E.P. : Y a-t-il alors un mode d'expression que vous préférez?}

A.J. : J'aime tout ce que je fais mais le mode qui demeure central pour moi, c'est la poésie. Le mode le plus affolant de tous et le plus amusant, c'est le cinéma qui est l'art le plus complet car il regroupe tout. C'est tous les arts réunis mais aussi le plus difficile car c'est un métier réalisé par des voleurs : le cinéma a toujours été un commerce, une industrie, de son développement aux États-Unis jusqu'à aujourd'hui. Or, l'art ne peut pas être industriel, c'est même une erreur de le considérer ainsi. Dans l'art, il y a la qualité et la quantité. La qualité, c'est le but, celle du produit que je fais. Comme le dit la Bhagavad-Gita ${ }^{4}$, "Pense à l'œuvre mais ne pense pas au fruit de l'œuvre $»^{5}$. Quel est le fruit de l'œuvre pour un artiste industriel ? C'est la gloire, l'argent et le pouvoir. Si on fait de l'art dans l'un de ces trois buts, alors ce ne sera pas un art véritable mais un art industriel. Il est nécessaire pour l'amusement, mais quel est le but de l'art? L'artiste, qui est en avance de quelques années sur la personne non artiste, n'est pas en avance pour être applaudi mais parce qu'il ouvre des frontières pour les autres : il te montre la beauté, la vérité et la bonté de l'être humain. Et tel est le but de l'art!

E.P. : Vous venez de terminer un film sur la psychomagie: pourquoi avoir voulu rendre compte de ces expériences?

A.J. : Parce que c'était nécessaire. Pour moi, la psychomagie, c'est l'art pour guérir. Notre société est malade. Nous vivons une période de double décadence : décadence de la spiritualité et décadence sociale, économique et écologique. Mais c'est comme un ver qui se retourne et duquel va naître un papillon : nous sommes à un moment d'écroulement et de changement. Auparavant, l'artiste était quelqu'un qui se détruisait et détruisait le monde : Charles Baudelaire, Franz Kafka, Arthur Rimbaud, autant de destructeurs que d'hommes détruits... Mais maintenant tout le monde connaît cette destruction, alors l'artiste doit apporter l'espoir et non ajouter à la décadence sa propre décadence. Personne aujourd'hui ne s'intéresse à ce qui m'arrive en tant qu'être humain : on est blasé, il y a trop de souffrance. La mission de l'artiste n'est pas de proposer des solutions mais de donner à concevoir positivement les choses. Cela ne veut pas dire qu'il faut faire de la littérature qui donne des conseils pour guérir, mais qu'il faut juste reconnaître la valeur de l'être humain et l'unité de tout. Être intelligent, c'est arriver au bonheur. Or, notre société ne développe que l'astuce.

Quand je faisais de l'art pour moi, j'étais astucieux et j'avais du succès. À la mort d'un de mes fils âgé de 24 ans, je me suis dit que j'allais faire un art qui servirait à quelque chose, un art qui puisse être utile. Dans Psychomagie, je montre comment guérissent des personnes. Ce film n'est pas un documentaire mais un film expérimental où, lorsqu'une personne vient me voir pour m'exposer un problème qui lui semble impossible à résoudre, je le dénoue. Il lui faut la foi, il faut qu'elle croie en moi. 
Ensuite la guérison, c'est commencer à guérir ; Comme pour l'alchimie, ce qui compte est le travail, le processus pour parvenir au but. Et tout cela ne fonctionne que par degrés.

E.P. : Vous employez souvent le tarot dans vos séances de psychomagie.

A.J. : Je pense qu'on ne se trouve qu'à partir de 50 ans. À cet âge, j'avais réalisé un film pour lequel on m'a escroqué, puisque l'on ne m'avait pas payé pendant un an. J'avais mon ex-femme, trois enfants, et pas un sou. Heureusement, j'avais un ami aux États-Unis qui était riche et qui m'a prêté 10000 francs, ce qui m'a permis de vivre à Joinville-le-Pont. Mais de quoi allais-je subsister au jour le jour? Je lisais le tarot, non pas pour voir le futur mais en tant que pratique artistique. Dans La Voie du tarot ${ }^{6}$, j'explique que c'est comme un test, comme une recherche pour moi. Je connaissais un médecin qui, pendant des années, m'envoyait chaque week-end quatre patients à qui je lisais le tarot gratuitement. J'investissais leur inconscient pour les aider à développer leur recherche. Quand j'étais dans la misère, j'ai vécu de séances de tarot pour lesquelles on me payait. Il y avait beaucoup de demandes car, après des années de pratiques bénévoles, j'ai reçu le bénéfice de ma générosité. Alors j'ai commencé à donner des cours gratuitement. Je faisais ça à la Maison des Mines à Paris, et pour en payer le loyer je faisais une collecte à la fin des séances en indiquant : « Tant que vous me donnez assez pour payer la salle, je continue ». Tous les mercredis, pendant une trentaine d'années, j'ai donné des conférences, puis j'ai gagné ma vie en assurant des cours. J'ai alors arrêté de faire payer les séances de tarot car il s'agit pour moi d'un travail sacré qui doit être gratuit. Il en est de même pour les actes de psychomagie : la thérapie doit être gratuite.

E.P. : Mais est-ce que dans ce dernier film, le fait qu'il y ait une caméra n'a pas changé la façon d'agir des consultants?

A.J. : Effectivement, c'est exactement le problème central que j'ai rencontré dans Psychomagie. Dans mes autres films, où je raconte une histoire, on a des rails, on a la caméra, on répète l'action, on la filme ; si ce n'est pas bien, on la filme de nouveau, on peut le faire vingt fois, jusqu'à ce qu'on obtienne la perfection. Pour Psychomagie, c'était différent : tu as une personne qui a un problème et tu n'as pas la moindre idée de la façon dont elle va réagir. Et elle non plus. C'est pourquoi la caméra doit être de taille réduite et la personne qui filme (c'est Pascale) se faire discrète pour ne pas se faire la remarquer. Elle est assise et tout à coup je lui dis : «Coupe la caméra ! - Oui, oui ", mais elle ne la coupe pas. Il m'arrive de lui demander de couper la caméra sans qu'elle le fasse. C'est un truc que j'utilise pour que la personne, qui pense ne plus être filmée, se comporte naturellement. Si cette personne sait qu'on la filme, rien ne se produit car, impressionnée par la caméra et pensant que ce qu'elle dit et fait est destiné à rester, elle n'est pas elle-même... Une guérison, à l'instar de la naissance d'un enfant ou la germination d'une graine, se fait dans l'obscurité. Si tu as un regard extérieur, elle ne se fait pas.

E.P. : Si nous revenons aux multiples modes d'expression de votre art, pouvez-vous nous parler des différentes techniques employées?

A.J. : En tant qu'artiste multiple, ce qui m'intéresse, ce sont justement ces différentes techniques. Le cinéma, encore aujourd'hui, n'est rien d'autre qu'une technique [un dispositif] pour un spectateur immobile qui, comme une rivière, reste essentiellement passif et se contente de recevoir. Il en est tout autrement de la bande dessinée qui, si elle parait être une sorte de petit cinéma, n'a en réalité rien à voir 
avec le 7e art : au cinéma tu dois filmer le mouvement en continuité alors que dans la bande dessinée, c'est ton cerveau qui doit créer le mouvement. Tu es une sorte de spectateur actif. Au cinéma, tu vois les choses les unes après les autres, alors que dans la bande dessinée chaque page cache la suivante qui révèle une inconnue. Quand on crée une bande dessinée, on doit être très attentif à chacune des pages et à la façon dont elles se succèdent. Pour le lecteur, c'est une découverte continuelle et cela exige qu'il soit honnête en ne feuilletant pas la BD. Au cinéma, c'est différent : le spectateur doit attendre pour voir la suite. Même sous forme de DVD, cette découverte est linéaire tandis que pour la BD, elle s'effectue par sauts. La bande dessinée me permet de gagner ma vie. En revanche, les huit films que j'ai réalisés ont été fait "gratuitement " parce tout le bénéfice m'a été volé. Faire un film ne permet pas de gagner le moindre sou car on te vole tout. On ne peut réaliser que par amour.

E.P. : Et le théâtre, quelle place a-t-il dans votre œuvre?

A.J. : Le théâtre est pour moi comme une religion. Il permet une chose merveilleuse : t'enlever du monde. En effet, lorsque tu rentres dans une salle de théatre, que tu y répètes, tu sors du monde. Le théâtre, c'est un monde dans le monde. À la différence de l'acteur de cinéma, le comédien de théâtre est comme un saint. Être acteur de théâtre doit être une vocation car c'est un métier très mal payé, où de plus le travail fourni s'efface. De nos jours, le théâtre est filmé, mis en DVD. Mais ça ne passe pas car une pièce théatre est faite pour être jouée devant un public vivant. C'est existentiel, héroïque. Cela n'a pas de durée, c'est là. Le théâtre est comme une messe, il est mystique.

E.P. : Et au théâtre justement, quelle place est accordée au spectateur, quel est son rôle ?

A.J. : J'ai commencé de façon normale, avec le spectateur d'un côté et la pièce de théâtre de l'autre. Par la suite, j'ai monté les pièces de lonesco et d'autres à mon goût, en séparant la littérature du théâtre. La littérature, c'est la base à partir de laquelle j'ai fait les décors, les costumes, les lumières, les actions, etc. Le théâtre n'est pas la pièce car celle-ci change selon la façon dont tu la montes. Peu à peu, j'ai commencé à intégrer le public dans la pièce de théâtre. Comment? En sortant du théâtre. J'ai écrit «Sortir le théâtre du théâtre » (" Sacar el teatro del teatro »), parce que le théâtre est un poncif : il y a une scène et un point de vue frontal du public, mais je peux faire du théâtre autour de personnes, dans un autobus... Puis j'ai éliminé la pièce de théâtre et les acteurs pour faire l'« acte théâtral », un acte donné à faire à une personne quelconque. Je disais à une personne : « J'ai tant d'argent pour toi, on va se présenter par exemple à l'école des Beaux-Arts: que veux-tu faire devant un public ? » Et ce n'était pas un public de théâtre mais des gens qui étaient là, les étudiants de l'université, et qui regardaient. J'ai commencé par aller à l'université d'architecture où il y avait mille étudiants. J'ai fait monter une vache sur scène, le cul vers eux, et j'ai montré dans une conférence que le cul de la vache avait la même structure qu'une cathédrale gothique (Rires). Le recteur est venu et m'a demandé de partir. Autre exemple : un étudiant en philosophie m'a dit: "Je voudrais avoir un faux ventre rempli d'œufs, être très bien habillé, en professeur d'université, avec cravate, et lire la Critique de la raison pure de Kant, tout en m'écrasant les œufs sur la tête », et je l'ai fait. Une danseuse m'a dit: "Je veux danser la mort du cygne en tutu, sans culotte, en faisant pipi sur scène pendant que je danse », et je l'ai fait. J'ai ainsi créé l'acte théâtral. Ce n'était que du théâtre - ce n'était pas de la guérison mais la libération des actes que ces personnes voulaient faire en public, pour casser les limites. Je suis ensuite passé aux happenings que j'ai appelés "éphémères paniques ». C'étaient des pièces de théâtre improvisées devant des gens et pour lesquelles j'ai éliminé tout le théâtre pour arriver à l'acte éphémère. Cela peut être des cris, des paroles, un millier de poulets dans le lieu... Le théâtre est conçu pour être répété chaque 
soir ; l'éphémère panique, on l'exécute une seule fois. On ne répète pas, on se met d'accord: "On va faire ça », et après on voit ce qui se passe.

E.P. : Qu'est-ce que le théâtre, et le happening particulièrement, a apporté aux autres modes d'expression?

A.J. : Après avoir monté une centaine de pièces de théâtre, je savais faire les décors, les costumes, et même la musique alors que je n'y connaissais rien. Maintenant, au cinéma je sais faire tout cela alors je peux diriger, vérifier les mouvements de caméra, la photographie, et j'accompagne le monteur une fois qu'on a filmé. Mon cinéma n'est pas commercial : dans un film commercial, chacun fait son œuvre et après ça s'unit. Je suis un poète qui exprime des choses personnelles. Je ne trouve pas de producteur mais des mécènes. Grâce au crowdfounding, les gens me donnent de l'argent, et j'ai ainsi découvert la façon d'être libre même financièrement. Dans le cinéma industriel, tu n'es pas libre car le producteur, qui est la personne la plus importante, décide de tout, et il s'agit de gagner le plus d'argent possible, ce qui implique de plaire au plus grand nombre de personnes. J'essaie aussi de ne pas travailler avec des stars: je prends mes fils car je cherche des personnes qui ne sont pas célèbres mais qui veulent jouer, en évitant les acteurs professionnels qui vont jouer comme des acteurs. J'ai fait Psychomagie parce que c'est un film sans acteurs. Ce sont des personnes réelles qui ont un problème et qui remplissent l'écran complètement, ce qui provoque une impression forte parce que c'est vrai. Dans un film, on est habitués à voir des mensonges car rien n'est vrai, les sentiments sont faux puisque ce sont des acteurs qui jouent. Alors j'ai éliminé les acteurs pour pouvoir arriver à un art libre. Je ne prétends pas à un grand public mais à un petit public qui aime. Il faut sacrifier beaucoup de choses, notamment le grand succès public, pour faire une œuvre qui soit utile.

Dans le cinéma, j'utilise beaucoup de moments improvisés. Dans Psychomagie, je montre que dans chacun de mes films, il y a des actes psychomagiques que personne ne voit. Dans La Danza de la realidad (2013), alors qu'un enfant a peur de l'obscurité, sa mère arrive et lui dit: «Tu n'auras plus peur de l'obscurité, tu vas devenir l'obscurité ». Elle le peint alors en noir et joue avec lui. J'ai ensuite appliqué cette méthode pour solutionner des problèmes que la psychanalyse met quatre ou cinq années à découvrir. La psychanalyse est un système qui dépend de la parole et où la personne est immobilisée. Ce ne sont pas des actes, ce sont des paroles. La psychomagie, ce sont des actes. On analyse l'arbre généalogique et on agit. Dans une guérison, il faut laisser la morale de côté ; il ne faut pas être immoral mais amoral pour pouvoir arriver à la solution d'un problème, parce qu'avec la moralité, il y a tout de suite la culpabilité, le péché.

Je pense qu'il y a un trésor intérieur dans chaque être humain : son cerveau. Mais on vit dans une cage créée par la famille, la société, la culture, l'histoire. On nous met dans une cage faite de préjugés. Pendant des siècles, on a cru à la logique aristotélicienne ${ }^{7}$ mais aujourd'hui on arrive à découvrir d'autres logiques, difficiles à comprendre pour nous mais néanmoins véridiques.

E.P. : Dans l'art aussi, il faut être amoral.

A.J. : Oui, tout à fait. Mais je me suis retiré de la politique et des religions, je ne touche pas à ça. L'art n'est pas politique, sinon tu limites ton public et tu n'apportes de solutions à personne. 
On apprend et au début on provoque des scandales. Au Mexique, on m'a dit qu'on ne pouvait pas faire un scandale à la télévision, ce à quoi j'ai répondu que c'était très facile et que je pouvais en faire un maintenant. Il y avait une Bible que j'ai posée par terre et dans laquelle j'ai commencé à donner des coups de pieds. Scandale! Une autre fois, j'ai détruit un piano. Dans une arène, le matador est un artiste et son instrument d'expression c'est le taureau qu'il finit par détruire. J'ai fait la même chose : j'ai produit des sons avec un piano que j'ai ensuite détruit, ce qui m'a pris une bonne heure car il n'est pas si facile de détruire un piano à coups de marteau. À la même heure, sur le canal principal de la télévision, il y avait un film de guerre qui avait beaucoup de succès auprès des Mexicains mais ils se sont téléphoné - " Il y a un fou qui détruit un piano!»-, et le scandale a été national. Là, j’ai pénétré dans la culture mexicaine d'un seul coup. Il s'agissait d'un scandale utile car c'était faire un parallèle avec la corrida, une tradition populaire. C'était donc bien une expression artistique que de détruire un piano.

E.P. : La réception de vos œuvres varie selon les pays. Comment l'expliquez-vous?

A.J. : Un pays, c'est un langage, et chaque langage a une philosophie différente. On ne pense pas pareil en anglais qu'en japonais ou en espagnol. Tout pays est un préjugé, une convention qui peut changer avec l'Histoire. Mais cela nous marque malgré tout. Moi, j'avais originairement un passeport chilien. À mon arrivée en France, j'ai eu un passeport français. Aujourd'hui, mon passeport chilien est périmé et je ne peux le refaire car il faudrait pour cela que je vive six mois au Chili. Je ne suis donc aujourd'hui plus chilien, mais un français... qui parle espagnol. C'est pourquoi je dis que ma patrie, ce sont mes chaussures: je suis sans nationalité.

E.P. : Est-ce que vous avez remarqué une influence du pays dans lequel vous vivez sur votre création?

A.J. : Quand tu es artiste, tout influence ta création. Tout artiste est un apprenti perpétuel. Au Mexique, à chaque fois que je faisais une pièce de théâtre, c'était un scandale épouvantable! Mais c'était sympathique. Aux États-Unis, c'était la recherche du dollar, tout le monde était fou avec le dollar. Ici, en France, je me trouve au calme. Les Français ne se rendent pas compte comme c'est idéalement calme par rapport à New York ou au Mexique. Même si Paris n'est plus le centre du monde, contrairement à ce qu'elle était quand je suis arrivé en 1953, la culture n'a pas changé de continent.

E.P. : Êtes-vous retourné au Mexique?

A.J. : Oui, quelques fois, pour présenter un film. Et puis, j'ai deux de mes fils qui y vivent. J'ai deux fils aussi qui y vivent. J'ai beaucoup lutté là-bas. Le Mexique a une qualité : les gens font un scandale dès que tu fais quelque chose qu'ils n'ont jamais vue et tu deviens alors l'ennemi public. Il y a eu un défilé de deux mille personnes contre moi, ce qui a été une expérience curieuse. Mais quand le temps passe et qu'ils s'habituent, tu deviens un héros. En ce moment, beaucoup de demandes me sont faites pour que je vienne vivre au Mexique : on m'a adopté, alors que j'ai dû m'en échapper! Un jour, on m'a dit: «Jodorowsky, on va te tuer ». Et c'était une vraie menace. On voulait me tuer parce qu'on m'accusait d'avoir insulté l'Église en raison $\mathrm{du}$ fait que j'avais fait passer un camion de corps nus devant un lieu de culte... On m'accusait d'avoir fait une messe noire alors que je cherchais juste une architecture ancienne pour mon film La Montaña sagrada. Il y a eu un scandale féroce. Le Mexique est un pays en proie à la violence mais, dans le même temps, il y a un sens de la fête qu'il n'y a pas en France. 
E.P. : Comment voyez-vous le rôle des nouvelles technologies, notamment d'Internet et des réseaux sociaux? Quelles nouvelles possibilités ouvrent-elles?

A.J. : Toutes les choses possèdent simultanément des parts de clarté et d'obscurité. Cela dépend de la façon dont elles sont utilisées. Tant qu'une technique est employée sans que ne soient développées les qualités humaines de ceux qui l'utilise, il y a catastrophe. Toute découverte technique doit être accompagnée d'une découverte intérieure. Les réseaux sociaux sont des royaumes des mots, et cela peut être très dangereux pour une société, comme une gangrène.

E.P. : Vous-même, vous utilisez beaucoup Facebook et Twitter.

A.J. : Evidemment. J'ai plusieurs millions de "suiveurs ». J'ai décidé d'employer ces outils car je me suis dit: "Le monde va mal, il faut changer le monde ». Je ne peux pas changer le monde mais je peux commencer par me changer moi-même. J'utilise Twitter et Facebook car tout ce que j'y écris peut être repris librement, il n'y a pas de droits d'auteur. Chaque jour, pendant une heure, j'écris donc gratuitement sur les réseaux sociaux où je fais cadeau, par exemple, de mes poésies. On me les vole, on me les prend. On apprend la générosité et la gratuité de la création. Je ne le fais pas dans le but d'obtenir quelque chose mais pour le seul plaisir de changer le monde. Aujourd'hui, j'ai 90 ans et suis près de cette mort que j'ai fini par accepter. Mais jusqu'au dernier moment, j'emploierai ma créativité pour changer le monde.

\section{NOTES}

1. Le "satori » est un mot japonais signifiant « compréhension ». Dans le bouddhisme Zen, cela désigne un soudain éveil spirituel qui révèle la véritable essence et l'unité de toute chose et de soi-même.

2. Le « koan » est une courte anecdote d'apparence absurde ou paradoxale se terminant souvent par une question que le maître bouddhiste pose au disciple. Il ne peut être résolu de manière intellectuelle mais fait appel à une forme de connaissance intuitive. La résolution $\mathrm{du}$ "koan » permet de progresser vers le «satori».

3. Il ne s'agit pas d'une guérison physique mais psychologique, ayant toutefois des répercutions concrètes sur la vie de la personne. En réalisant des actes symboliques, celle-ci devient actrice de sa guérison et cela permet la résolution d'un mal-être intérieur. Jodorowsky parle aussi dans ce cas du « théâtre de la guérison ».

4. La Bhagavad-Gita est la partie centrale du poème épique Mahabharata, un des écrits fondamentaux de l'hindouisme.

5. La citation exacte de la Bhagavad-Gita (traduction de Camille Rao et Jean Herbert, d'après la traduction anglaise de Shrî Aurobindo, éd. Librairie d'Amérique et d'Orient Adrien Maisonneuve, 1991) est : « Tu as droit à l'action, mais seulement à l'action, et jamais à ses fruits ; que les fruits de tes actions ne soient point ton mobile; et pourtant ne permets en toi aucun attachement à l'inaction » (chapitre 2, verset 47).

6. Alejandro Jodorowsky, Marianne Costa, La Voie du tarot, Paris, Albin Michel, 2004. 
7. Également appelée «logique formelle », la logique aristotélicienne traite de la forme des raisonnements et non des contenus empiriques sur lesquels ces raisonnements portent. Le célèbre « syllogisme » constitue la forme pure de cette logique.

\section{RÉSUMÉS}

Ce texte est la transcription d'une conversation avec Alejandro Jodorowsky à propos de l'ensemble de son œuvre et des différents arts qu'il pratique. Le cinéaste aborde tour à tour le théâtre, le cinéma, la psychomagie, la bande dessinée et met en valeur à la fois les liens entre ces différents modes d'expression et leurs caractéristiques propres. En retraçant son parcours, Alejandro Jodorowsky nous fait partager sa vision de l'art, de l'artiste et son rapport au monde.

This text gives an account of a conversation with Alejandro Jodorowsky about his work as a whole and the different arts he practises. The filmmaker approaches successively theatre, cinema, psychomagic, comics and highlights the links between these different modes of expression and their own characteristics. In retracing his career, Alejandro Jodorowsky shares his vision of art, the artist and his relationship with the world.

\section{INDEX}

Mots-clés : Alejandro Jodorowsky, entretien, psychomagie, inconscient, intermédialité

Keywords : Alejandro Jodorowsky, interview, psychomagic, subconscious, intermediality

\section{AUTEUR}

\section{ELISABETH POUILLY}

Elisabeth Pouilly est docteure en Études Théâtrales. Elle a soutenu sa thèse sur « L'état d'esprit performatif" dans le théâtre et le cinéma d'Alejandro Jodorowsky » à l'Université Paris III Sorbonne Nouvelle, sous la direction de Joseph Danan. Elle a publié des articles sur les éphémères paniques, sur les liens entre le groupe Panique et Dada, sur les Midnight movies, et un article dans la revue d'art contemporain Marges traitant de l'expérience psychomagique de Jodorowsky. 